\title{
The Reflective Property of a Parabola
}

\author{
FINBARR HOLLAND
}

\section{INTRODUCTION}

Thanks to my Mathematics teacher, An tUasal Concubhair ÓCaoimh, I left school knowing some of the properties of the conics. However, it wasn't until much later that I came to appreciate their relevance in our daily lives. Ironically, when Mathematics teachers are nowadays expected to teach real-world applications of their subject, and we're surrounded by those of the parabola especially, and, to a lesser extend, by those of the ellipse, conics have no place in the school curriculum. It is this irony which prompted this note, whose purpose is to remind readers of the reflection property possessed by the parabola, and to point out that no other curve shares this feature, something that may not be widely known.

\section{A Reflection Property of the Parabola}

Recall that a parabola is a set of points that are equidistant from a fixed point (called its focus), and a fixed line (called its directrix). We may suppose, for definiteness, that the focus is $(a, 0)$, where $a>0$, and that the directrix has equation $x+a=0$. Then, in order that the point $(x, y)$ be on the resulting parabola, it is necessary and sufficient that

$$
\sqrt{(x-a)^{2}+y^{2}}=|x+a|
$$

i.e., that $y^{2}=4 a x$. This is the equation of the parabola with which we'll work from now on.

The following is a basic property of any parabola, and is the key idea behind such everyday objects as lamp-shades, satellite dishes, car headlamps and hearing aids.

Theorem 1. Let $P=\left(a t^{2}, 2 a t\right)$ be any point on the parabola $y^{2}=$ $4 a x$, and let $F=(a, 0)$. Let the tangent to the parabola at $P$ meet the $x$-axis at $Q$. Then $|P F|=|F Q|$. 
Proof. If $t=0, P$ coincides with the origin and the result is clear. So, suppose $t \neq 0$. Then $y=x / t+a t$ is the equation of the tangent at $P$. This intersects the $x$-axis at $Q=\left(-a t^{2}, 0\right)$. Hence

$$
|Q F|=\left|-a t^{2}-a\right|=a\left(1+t^{2}\right) \text {. }
$$

But,

$$
\begin{aligned}
|F P| & =\sqrt{\left(a t^{2}-a\right)^{2}+(2 a t-0)^{2}} \\
& =\sqrt{\left(a^{2} t^{4}-2 a^{2} t^{2}+a^{2}+4 a^{2} t^{2}\right.} \\
& =\sqrt{\left(a^{2} t^{4}+2 a^{2} t^{2}+a^{2}\right.} \\
& =\sqrt{\left(a t^{2}+a\right)^{2}} \\
& =a\left(1+t^{2}\right) .
\end{aligned}
$$

Thus, $|P F|=|F Q|$.

Corollary 1. $\angle F P Q=\angle P Q F$.

This means that the tangent at any point $P$ of a parabola is equally inclined to the line joining $P$ to its focus and the axis of the parabola. As a consequence, Heron's Reflection Principle comes into play, and we have the following fundamental fact: a light ray travelling towards a parabola from right to left along a straight line parallel to the axis of the parabola is reflected through its focus. Conversely, a light ray emitted from a source situated at the focus of a parabola is reflected by the parabola along a line parallel to its axis.

Shouldn't this simple fact be taught in school?

\section{This Reflection Property Characterizes the Parabola}

Could a satellite dish have been invented without knowing the properties of the parabola that the Greeks discovered over two thousand years ago?

If we think of such a dish as being a surface of revolution obtained by rotating a smooth function about the positive $x$-axis, this question is answered by the following result.

Theorem 2. Suppose $a>0, f$ is continuous on $[0, \infty)$, with $f(0)=$ 0 and $f(a) \neq 0$, and differentiable on $(0, \infty)$, with $f^{\prime} \neq 0$ there. Suppose, in addition, that the tangent to any point $P \in f$ is equally inclined to the line joining $P$ and $(a, 0)$, and the $x$-axis. Then

$$
f^{2}(x)=4 a x, \forall x \in[0, \infty) .
$$


Proof. Let $t>0$. The tangent at $(t, f(t))$ has equation $y-f(t)=$ $f^{\prime}(t)(x-t)$. This meets the $x$-axis at $\left(t-\frac{f(t)}{f^{\prime}(t)}, 0\right)$. By hypothesis, the distance between this point and $(a, 0)$ is the same as the distance between the latter and $(t, f(t))$. Thus

$$
\left|t-a-\frac{f(t)}{f^{\prime}(t)}\right|=\sqrt{(t-a)^{2}+f^{2}(t)} .
$$

Equivalently

$$
-2 \frac{(t-a) f(t)}{f^{\prime}(t)}+\frac{f^{2}(t)}{f^{\prime 2}(t)}=f^{2}(t)
$$

In other words, for all $t>0$,

$$
\begin{aligned}
f^{2}(t)=f^{2}(t) f^{\prime 2}(t)+2(t-a) f(t) f^{\prime}(t), & \\
& f^{2}(t)+(t-a)^{2}=\left(f(t) f^{\prime}(t)+(t-a)\right)^{2} .
\end{aligned}
$$

Let

$$
g(t)=f^{2}(t)+(t-a)^{2}, \quad t \geq 0,
$$

so that $g^{\prime}(t)=2\left(f(t) f^{\prime}(t)+(t-a)\right), t>0$. We deduce that $g$ satisfies the differential equation

$$
g^{\prime 2}(t)=4 g(t), 0<t<\infty, g(0)=a^{2} .
$$

However, $g>0$, and so $h=\sqrt{g}$ is differentiable on $(0, \infty)$, and satisfies the simpler non-linear differential equation

$$
h^{\prime 2}(t)=1,0<t<\infty, h(0)=a .
$$

But $h^{\prime 2}-1=\left(h^{\prime}-1\right)\left(h^{\prime}+1\right)$, and so, for each $t>0$, either $h^{\prime}(t)=1$ or $h^{\prime}(t)=-1$. Since every derivative satisfies the Intermediate Value Property, this means that if $h^{\prime}$ takes the values -1 and +1 , it must, perforce, take the value 0 , which it patently does not! Conclusion: either $h^{\prime} \equiv 1$ or $h^{\prime} \equiv-1$. In other words, for all $t \geq 0$, either $h(t)=$ $t+a$, or $h(t)=-t+a$, i.e., either $g(t)=(t+a)^{2}$ or $g(t)=(-t+a)^{2}$. Thus,

$$
f^{2}(t)+(t-a)^{2}=(t+a)^{2} \text {, or } f^{2}(t)+(t-a)^{2}=(t-a)^{2} .
$$

The second of these possibilities is plainly untenable. Hence $f^{2}(t)=$ 4at, $\forall t \geq 0$. In other words, $f$ is the upper or lower branch of the parabola $y^{2}=4 a x$. 
Finbarr Holland,

School of Mathematical Sciences,

University College Cork,

Cork, Ireland

f.holland@ucc.ie

Received on 13 October 2010. 